\title{
Characterization of Mesoscale Waves in the Jupiter NEB by Jupiter InfraRed Auroral Mapper on board Juno
}

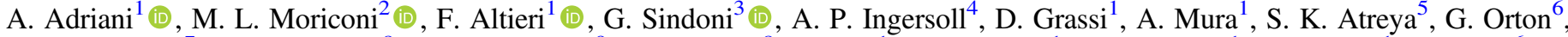 \\ J. I. Lunine ${ }^{7}$, L. N. Fletcher ${ }^{8}$ (D), A. A. Simon ${ }^{9}$ (D), H. Melin ${ }^{8}$, F. Tosi ${ }^{1}$, A. Cicchetti ${ }^{1}$, R. Noschese ${ }^{1}$, R. Sordini ${ }^{1}$, S. Levin ${ }^{6}$, \\ J. Bolton ${ }^{10}$, C. Plainaki ${ }^{3}$, and A. Olivieri ${ }^{3}$ \\ ${ }_{1}^{1}$ INAF-Istituto di Astrofisica e Planetologia Spaziali, Via Fosso del Cavaliere 100, 00133 Roma, Italy \\ ${ }^{2}$ CNR-Istituto di Scienze dell'Atmosfera e del Clima, Via Fosso del Cavaliere 100, 00133 Roma, Italy \\ Agenzia Spaziale Italiana, Via del Politecnico snc, 00133 Roma, Italy \\ ${ }^{4}$ California Institute of Technology, 1200 E. California Blvd., Pasadena, CA 91125 USA \\ ${ }^{5}$ University of Michigan, 500 S. State Street, Ann Arbor, MI 48109 USA \\ ${ }^{6}$ Jet Propulsion Laboratory, California Institute of Technology, 4800 Oak Grove Drive Pasadena, CA 91109 USA \\ ${ }^{7}$ Cornell University, 410, Thurston Avenue, NY 14850-2488 USA \\ ${ }^{8}$ Department of Physics and Astronomy, University of Leicester, Leicester, UK \\ ${ }^{9}$ NASA Goddard Space Flight Center, 8800 Greenbelt Road, Greenbelt, MD 20771 USA \\ ${ }^{10}$ Southwest Research Institute, 6220 Culebra Road, San Antonio, TX 78238 USA \\ Received 2018 May 16; revised 2018 September 18; accepted 2018 September 26; published 2018 November 2
}

\begin{abstract}
In 2017, the Jupiter InfraRed Auroral Mapper (JIRAM), on board the NASA-ASI Juno mission, observed a wide longitude region $\left(50^{\circ} \mathrm{W}-80^{\circ} \mathrm{E}\right.$ in System III) that was perturbed by a wave pattern centered at $15^{\circ} \mathrm{N}$ in the Jupiter's North Equatorial Belt (NEB). We analyzed JIRAM data acquired on 2017 July 10 using the M-channel and on 2017 February 2 with the spectrometer. The two observations occurred at different times and at slightly different latitudes. The waves appear as clouds blocking the deeper thermal emission. The wave crests are oriented north-south, and the typical wave packet contains 10 crests and 10 troughs. We used Fourier analysis to rigorously determine the wavenumbers associated with the observed patterns at a confidence level of $90 \%$. Wavelet analysis was also used to constrain the spatial localization of the largest energies involved in the process and determine the wavelengths carrying the major contribution. We found wavelengths ranging from 1400 to $1900 \mathrm{~km}$, and generally decreasing toward the west. Where possible, we also computed a vertical location of the cloud pressure levels from the inversion of the spectral radiances measured by the JIRAM spectrometer. The waves were detected at pressure levels consistent with the $\mathrm{NH}_{3}$ as well as $\mathrm{NH}_{4} \mathrm{SH}$ clouds. Phase velocities could not be determined with sufficient confidence to discriminate whether the alternating crests and troughs are a propagating wave or a manifestation of a fluid dynamical instability.
\end{abstract}

Key words: planets and satellites: atmospheres - planets and satellites: gaseous planets - waves

\section{Introduction}

Mesoscale atmospheric phenomena refer to horizontal motion scales ranging from $\sim 10$ up to $1000 \mathrm{~km}$ on the Earth (Lin 2007). On Jupiter, the same definition, re-scaled for the planet's larger size, can be reasonably applied to those phenomena whose dimensions range from $\sim 100$ up to $10000 \mathrm{~km}$. Mesoscale waves, as revealed by cloud patterns, have been observed on Jupiter since the 1980s. Voyager-ISS (Hunt \& Muller 1979; Flasar \& Gierasch 1986), New Horizon with its Multispectral Visible Imaging Camera (MVIC) and its Long Range Reconnaissance Imager (LORRI; Simon et al. 2015a), and Galileo-NIMS (Arregi et al. 2009) all detected wavy features on the underlying clouds at wavelengths ranging from the violet $(416 \mathrm{~nm})$ to the NIR $(975 \mathrm{~nm})$. All these wavelengths found similar pressure levels no deeper than $0.8 \mathrm{bar}$, corresponding to the upper troposphere, and where the ammonia cloud deck is predicted to occur (West et al. 2004; Atreya et al. 2005). The studies carried out on these data all converged on wave crests oriented northsouth and horizontal wavelength close to $300 \mathrm{~km}$. More recently, larger-scale waves $(\sim 1200 \mathrm{~km})$ have been seen by Hubble with its Wide Field Camera 3 (WFC3; Simon et al. 2015b). In a few cases, the phase speeds could be evaluated as well, giving values $\sim 90 \mathrm{~m} \mathrm{~s}^{-1}$ faster than the equatorial zonal wind speed (Simon et al. 2015b). All of the above studies are limited to the visible region of the spectrum, suggesting that the observed waves were modulating the $\mathrm{NH}_{3}$ ice clouds or the upper tropospheric hazes. Neither Voyager-IRIS, nor Galileo-NIMS, nor Cassini VIMS captured wave trains with their 4-5 $\mu \mathrm{m}$ channels, which were able to sound down to the $\mathrm{NH}_{4} \mathrm{SH}$ cloud level, possibly because their spatial resolution was much too low to do so. A contraction and expansion of the NEB has been revealed by ground-based observations (Fletcher et al. 2017) starting from 2016-17. However, neither the COMICS instrument (Kataza et al. 2000), on the Subaru Telescope, nor the VISIR instrument (Lagage et al. 2004) on ESO's Very Large Telescope, can sound down to the $\mathrm{NH}_{4} \mathrm{SH}$ cloud level with their spectral ranges $(8-25 \mu \mathrm{m})$; thus, their findings pertain only to the upper troposphere and stratosphere. Spectra of Jupiter at $5 \mu \mathrm{m}$ were acquired using NIRSPEC on the Keck II telescope (Bjoraker et al. 2015), allowing determination of the cloud structure in the 2-6 bar range of pressure levels at different latitudes. The longitude coverage in this case was not sufficient to identify any wave patterns.

Jupiter InfraRed Auroral Mapper (JIRAM) observed wavy cloud features along the NEB by its M-band $(5 \mu \mathrm{m})$ imager (hereafter IMG-M) on more than one occasion (Fletcher et al. 2018). Visually, the acquired images show the crests occurring every $1^{\circ}-2^{\circ}$ longitude degrees. Similar patterns also have been observed during the Juno mission by Hubble Space Telescope's WFC3, NIRI (Gemini North Telescope) and The 
Very Large Telescope VISIR, the latter with a new capability allowing it to provide improved $5 \mu \mathrm{m}$ diffraction-limited images. The non-Juno results are reported in two companion papers (Fletcher et al. 2018; Simon et al. 2018). The JIRAM working range, both in the imager and in the spectroscopic channels, covers the $2-5 \mu \mathrm{m}$ spectral region, therefore allowing it to sound levels deeper than the 1-bar pressure level, with a spatial detail that can reach a few tenths of a kilometer. Grassi et al. (2017) report a 5-bar sensitivity depth for clear sky conditions (hot spot), on the basis of the retrieved $\mathrm{NH}_{3}$ mixing ratio values. In this paper, we aim to characterize the horizontal and vertical structure of similar wavy cloud features. In Section 2, we describe the JIRAM instrument, the data sets used for the analyses, and the pre-processing techniques applied to the images. In Section 3, the signal selection and processing methods applied to the M-channel data are described. In Section 4, we trace out a summary of the retrieval calculation applied to the spectrometer data. In Section 5, the results of the two different analyses carried out on the imager and spectrometer data are given, and limits and implications are discussed in Section 6. Finally, we summarize the principal results achieved in this study in the summary and conclusions (Section 7).

\section{Observations}

JIRAM (Adriani et al. 2014) is composed of a spectrometer and an imager, sharing the same telescope. The spectrometer covers the $2-5 \mu \mathrm{m}$ range with an average spectral sampling of $9 \mathrm{~nm} /$ band (average spectral resolution of $15 \mathrm{~nm}$ ) and simultaneously acquires 336 spectra, arranged along a line of spatially contiguous pixels (slit). JIRAM spectra are combined with context images, acquired by the imager channel, whose focal plane is divided into sub-planes, integrating the incoming radiance over a broad spectral range centered around $4.78 \mu \mathrm{m}$ (IMG-M) or $3.46 \mu \mathrm{m}$ (IMG-L), the latter channel having been designed for monitoring the aurora $\mathrm{H}_{3}^{+}$emissions. Both imager sub-planes have a size of 432 by 128 pixels and the field of view (FOV) of individual pixels is about $240 \mu \mathrm{rad}$ for both spectrometer and imager. Geometric information was obtained by using algorithms based on the NAIF-SPICE tool (Acton 1996; Acton et al. 2018) for each image of the spectrometer and imager channels. JIRAM raw data are radiometrically calibrated in units of spectral radiance $\left(\mathrm{W} / \mathrm{m}^{2} \mu \mathrm{m} \mathrm{sr}\right)$ as described by Adriani et al. (2014).

JIRAM targeted the Jupiter equatorial region during all the Juno pericenter ("perijove" or PJ) passages. The IMG-M captured wave-like cloud patterns on more than one occasion, but only occasionally in favorable viewing conditions. In a few cases, the spectrometer slit also obtained a suitable coverage of the region of interest for coupled imaging/spectral studies. Here we analyze the 2017 July 10th data set (PJ7), where the whole wave pattern has been captured from the IMG-M, but with a single overlapping spectroscopy slit, and the 2017 February 2nd (PJ4) data set, where only a portion of a wave that was a few degrees south of the PJ7 one has been observed, but with many spectral slits. Both data sets were acquired with Juno approaching the planet night side, adjacent to the terminator region. In the PJ7 occurrence, a long mesoscale wave has been imaged in the $\mathrm{NEB}$, in three successive sequences of measurements, acquired at time intervals of $\sim 70$ minutes (Figure 1(a)). These data are characterized by emission angles in the range $1^{\circ}-60^{\circ}$ and spatial resolutions starting from $\sim 240 \mathrm{~km} \mathrm{pixel}^{-1}$ (farthest spacecraft position)_for the image acquired at 08:35 UTC-up to $\sim 220 \mathrm{~km}$ pixel $^{-1}$ - for the image acquired at 10:55 UTC. The FOVs of these images partially overlap with each other, and some portion of the wave is replicated under different viewing angles. The same region was probed about 10 hours later, with the spacecraft closer to the planet. In this second sequence, the data are characterized by emission angles in the range $5^{\circ}-40^{\circ}$ and spatial resolutions starting from $\sim 125 \mathrm{~km} \mathrm{pixel}^{-1}$, for the image acquired at 18:42 UTC, up to $\sim 110 \mathrm{~km} \mathrm{pixel}^{-1}$, for the image acquired at 20:01 UTC (Figure 1(b)). The data in Figure 1 have been corrected for the proper emission angle to compensate for the radiance values off-boresight. We used these data sets to evaluate the horizontal wavelength of the wave train and to give a first estimation of the pressure level where the clouds are shaped by this mesoscale wave pattern. The PJ4 occurrence allowed us to characterize the effect of the waves on the cloud distributions and to provide further information about the estimated pressure level via the spectrometer data analysis.

\section{IMG-M Data Analysis}

The wave pattern is visible in all three left panels of Figure 1, approximately at the northern limit of the NEB at its boundary with the Northern Tropical Zone. The pattern can be seen in greater detail in the right panels of the same figure, acquired about 10 hours later. The propagation direction of the wave looks aligned along the zonal flow and the crests centered around $\sim 15^{\circ} \mathrm{N}$ planetocentric latitude. It appears as a series of bands (wave fronts) of low (dark) and high (bright) radiance, approximately transverse to the latitude circles and overlying different cloud patterns, resembling laminar flow structures. The wave fronts are not perfectly aligned in the north-south direction and the whole wave train is not uniform in its meridional dimension. We evaluate the wavelength of the wave pattern by applying a spectral analysis to the profiles of radiances extracted from the $15^{\circ} \mathrm{N}$ latitude positions. The profiles, approximately centered on the wave fronts of Figure 1, have been binned to a regular step of 0.4 degrees to get samples uniformly spaced in longitude. This pre-processing operation is necessary to apply standard Fourier and wavelet transforms to the space samples.

In order to perform the spatial analysis, we used both Fourier transform (Bendat \& Piersol 1986) and the wavelet transform methods (Graps 1995; Resnikoff \& Wells 1998). Fourier transform is a powerful tool for analyzing the components of stationary signals, while the wavelet transform method also allows analysis of components of non-stationary signals. In other words, they are well-suited to identify possible changes in wave amplitudes and/or wavenumber/frequency related to physical mechanisms (for example, wave-wave or wave-turbulence interactions). Moreover, the Fourier method allows us to search for the highest wavenumber contributions, whereas we use wavelets principally to determine the spatial characteristics and possibly breaking or interaction areas of the imaged mesoscale waves. Finally, the wavelengths of the waves are converted from degrees to kilometers (see the Appendix for more details).

\section{Spectral Data Analysis}

In order to retrieve the cloud properties of the waves, we require the full spectral capability of JIRAM. Although the JIRAM IMG-M covered the NEB with a high accuracy, we 
(a)
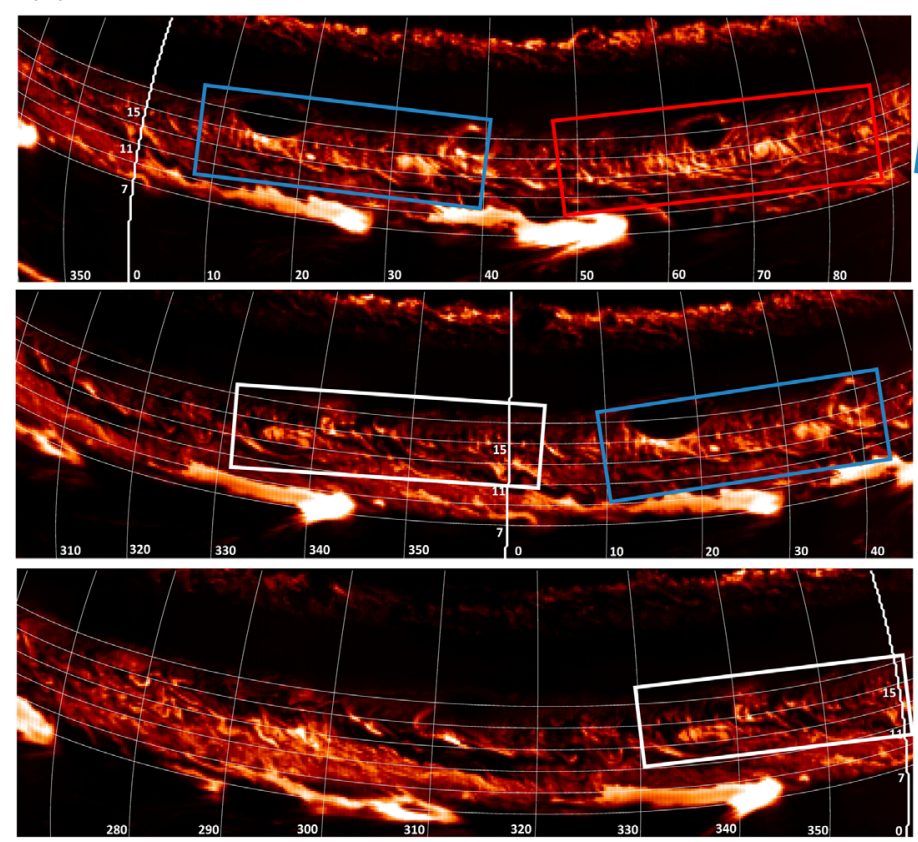

(b)
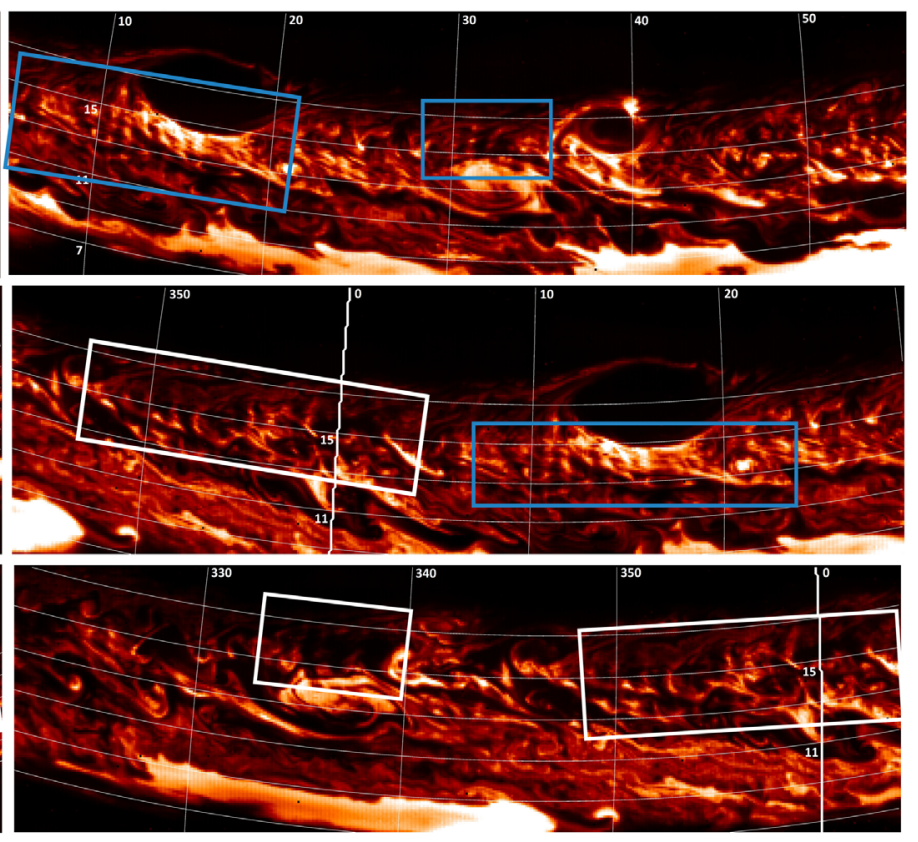

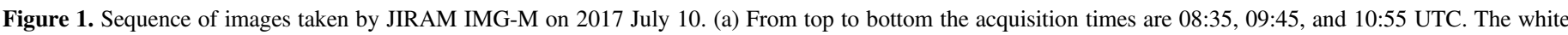

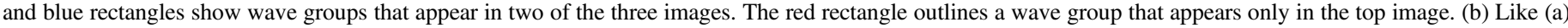
but for the acquisitions of 18:42, 19:22 and 20:01 UTC. All the images are in planetocentric coordinates and have been corrected for the emission angle.

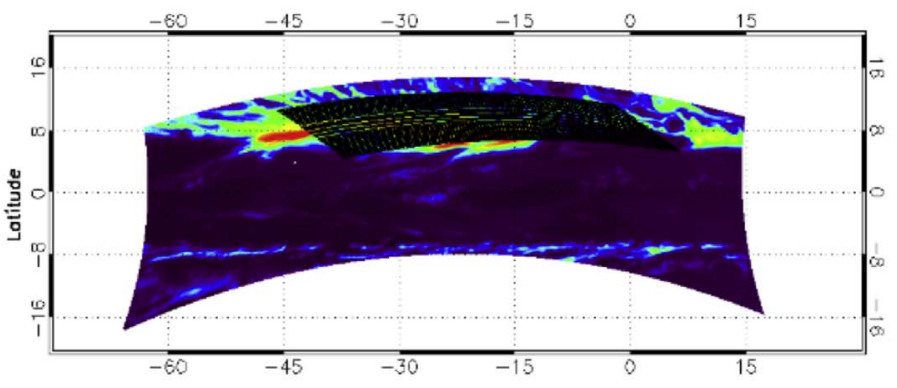

System III Eost Longitude

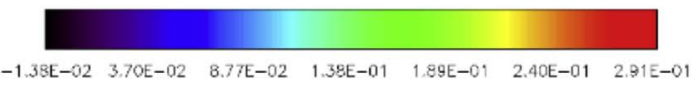

Radiance $\left[w /\left(\mathrm{m}^{2} \mathrm{str}\right)\right]$

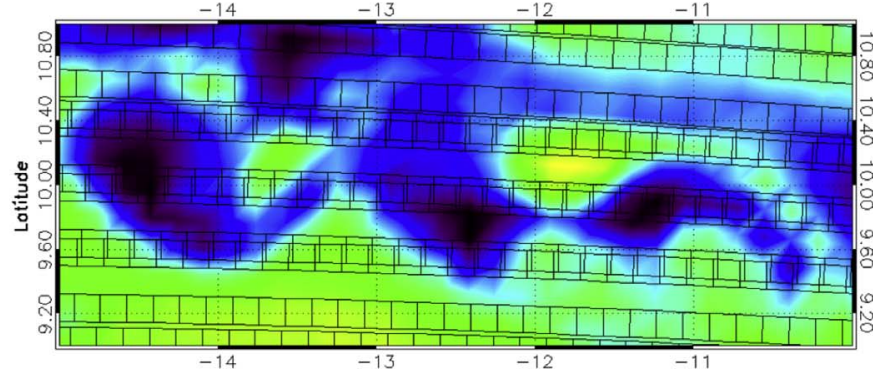

System III Eost Longitude

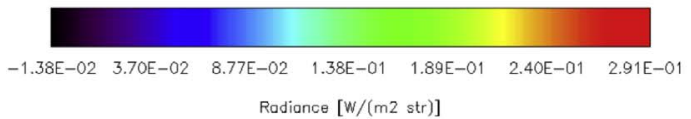

Radiance $[\mathrm{W} /(\mathrm{m} 2 \mathrm{str})]$

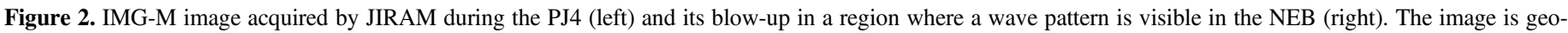

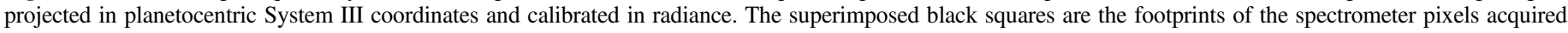
during the same measurement session.

have very limited coverage for the spectrometer during PJ7. However, we analyzed another case study, observed during PJ4, where the coverage of the spectrometer was more complete. Figure 2 shows the IMG-M acquisition taken on 2017 February 2nd where a wavy atmospheric structure was visible. In particular, we observed a clear wave pattern in the region between $-15^{\circ}$ and $-10^{\circ}$ in East longitude and $9^{\circ}-11^{\circ} \mathrm{N}$ in latitude (Figure 2). However, at lower latitudes than the PJ7 waves, the features appear visually similar enough for comparison of their properties.

Many spectrometer slit acquisitions are required to give good geographical coverage. The good agreement between the IMG-M and the spectrometer (SPE) data as regards location and brightness of the feature was confirmed by integrating the SPE radiance in each pixel at the same wavelengths as in IMG-M and superimposing the two geo-projected images (Figure 3). The slight shift we observe between the SPE and the IMG-M is due to the slight difference in latitude between the two slits together with the oblique position of the wave crests. Moreover, the intensity of the radiation coming from the IMG-M is higher than the one from the SPE because of the different integration spectral range of the two JIRAM channels. However, in both profiles we observe a mean variation in terms of thermal brightness of the order of about $7 \%-8 \%$ for longitudes between $15^{\circ}$ and $-10^{\circ}$, corresponding to the mean maximum thermal brightness for IMG-M around $243 \mathrm{~K}\left(0.20 \mathrm{~W} \mathrm{~m}^{-2} / \mathrm{sr}\right)$ and the mean minimum around 
JIRAM IMG M filter - 170202_005100_JUNO_IMG_00
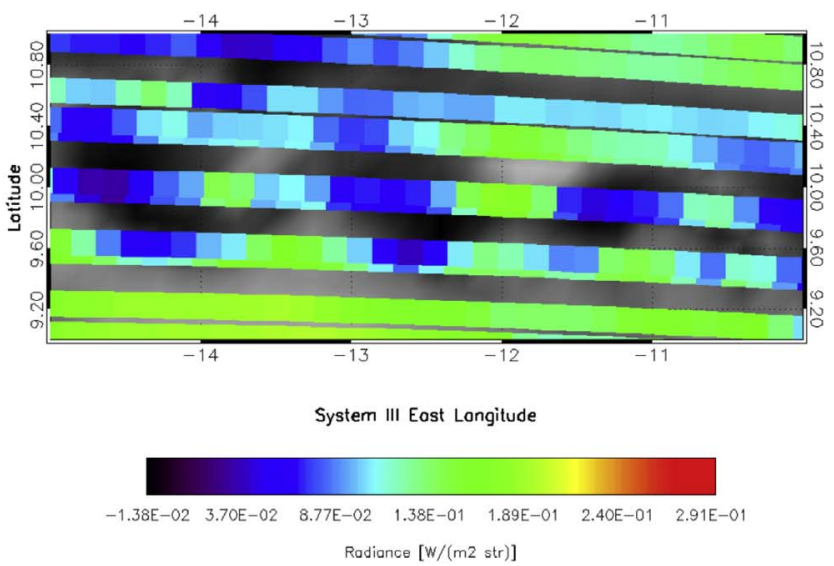

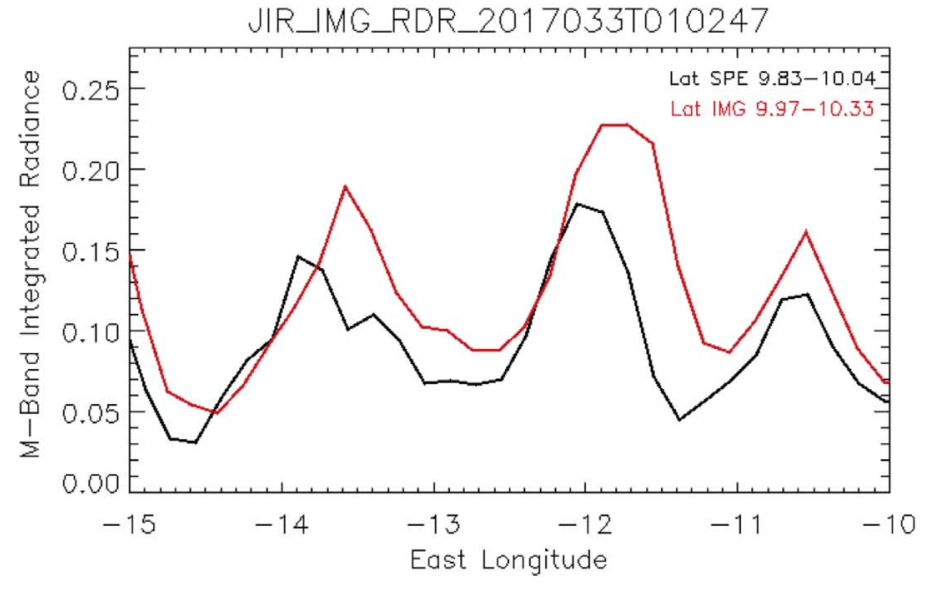

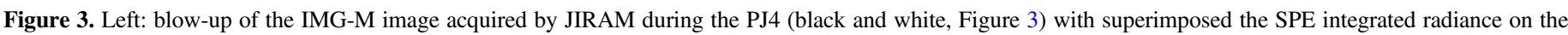

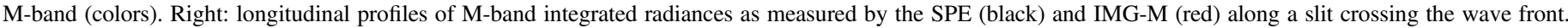

$427 \mathrm{~K}\left(0.08 \mathrm{~W} \mathrm{~m}^{-2} / \mathrm{sr}\right)$. Whereas for SPE we have, respectively, $238 \mathrm{~K}\left(0.15 \mathrm{~W} \mathrm{~m}^{-2} / \mathrm{sr}\right)$ and $220 \mathrm{~K}\left(0.05 \mathrm{~W} \mathrm{~m}^{-2} / \mathrm{sr}\right)$. The intensity of the observed variations is slightly lower in comparison with those found in the wave patterns studied by Fletcher et al. (2018), of the order of $10 \%$.

The retrieval procedure, specifically developed for JIRAM data, has already been successfully used for the characterization of the clouds structures in the white ovals of Jupiter's southern hemisphere (Sindoni et al. 2017). The data inversion technique is based on the Bayesian approach (Rodgers 2000) and uses the Gauss-Newton iterative procedure to minimize the $\chi^{2}$ function. The synthetic spectrum used in the inversion algorithm takes into account multiple scatterings, both by molecules and particles, and it is computed by using a line-by-line code in the plane-parallel approximation (Ignatiev et al. 2005). Since the current Juno orbits allow JIRAM to observe mainly during the night side, and the spectrometer measurements we selected have solar zenith angles higher than $90^{\circ}$, we adapted the retrieval procedure, originally developed for the analysis of the reflected sunlight in the $2.4-3 \mu \mathrm{m}$ range by Sindoni et al. (2017), for the exploitation of the thermal-dominated spectral range at $4.5-5 \mu \mathrm{m}$. An example of the best fit in this spectral range is shown in Figure 4. Some narrow features are not well fit as our retrieval scheme considers fixed values for the gaseous component in $\mathrm{PH}_{3}$ and $\mathrm{H}_{2} \mathrm{O}$ and does not consider arsine, but these do not affect the cloud-property retrieval we are interested in (see the Appendix for details about the retrieval method).

\section{Results}

\subsection{Profile Extraction and Signal Processing from PJ7 Data}

The two data sequences acquired at PJ7 and described in Section 3 will hereafter be called SS1 and SS2 (Figure 1 column (a) and column (b), respectively). SS1 and SS2 refer to a common region observed within $10 \mathrm{hr}$ time difference. Inside each sequence, the sample records partially overlap in longitude. These spatial series have been investigated by means of Fourier and wavelet transforms to identify, among the prominent wave signals in wavenumber space, the spectral band of monochromatic waves associated with the pattern imaged in Figure 1. Both spectral analyses have been applied to

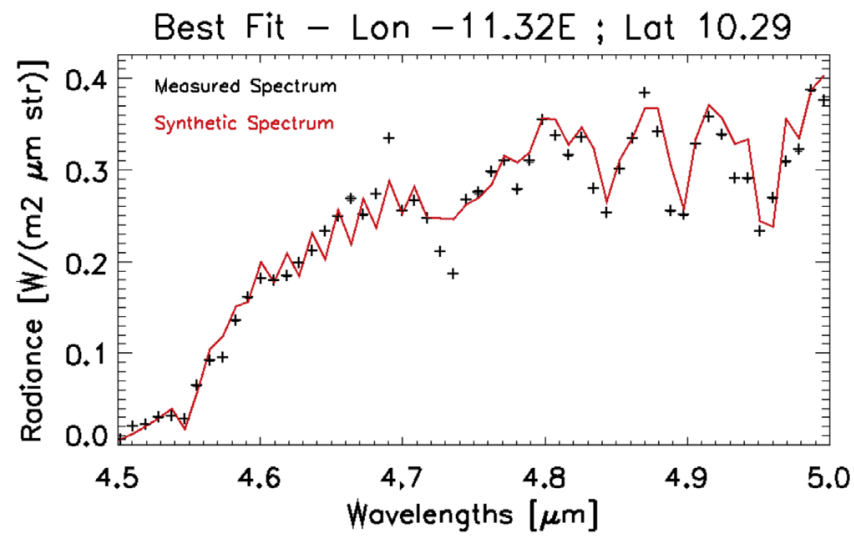

Figure 4. Example of spectral best fit for a pixel acquired pointing a longitude of $-11.32 \mathrm{E}$ and a latitude of 10.29 . The black points and the red line represent the JIRAM data and the synthetic spectrum, respectively.

the signals to understand how much is due to stationary or nonstationary contributions and where the breaking is occurring. In wavenumber space we cut off all the contributions less than 0.5 (wavelength $>2500 \mathrm{~km}$ ), according to the visual inspection of the waves in Figure 1, as irrelevant to the present analysis. Therefore, we applied a high-pass filter, with cutoff at 0.5 , corresponding approximately to a spatial wavelength of two longitude degrees. In the Appendix the wavelet transforms of the signals before and after the filtering are shown (Figure 10).

The unfiltered data clearly show the prominence of long period signals (period $>10^{\circ}$ longitude) whereas the filtered ones emphasize the presence of a quasi-continuous process, with periods in the range 0.8-2.5 degrees of longitude ( 1000-3000 km).

The Fourier analysis of the filtered signals permitted us to determine the principal wavenumber peaks and their significance level. The significance level is fixed on the basis of a mean background spectrum around which the potential spectra of the geophysical process can be randomly distributed. We used these spectra to establish a null hypothesis for the significance of a peak in the power spectrum (Torrence \& Compo 1998). Briefly, the signal is considered affected by two possible background noises: white noise (background power independent from the frequency/wavenumber) or red noise 

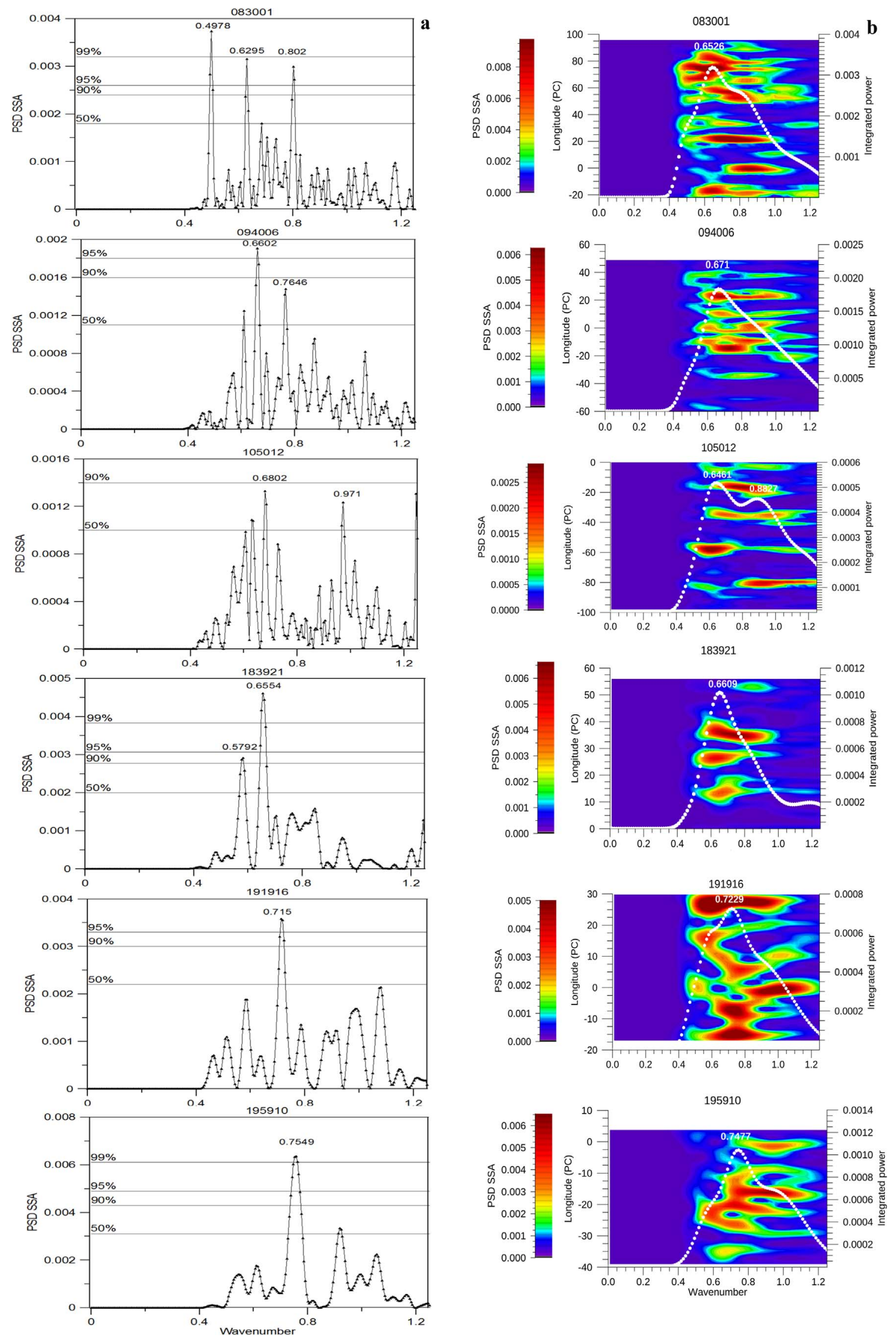

Figure 5. Power spectra of the Fourier and wavelet transforms of the filtered signal. From top to bottom, the plots refer to SS1 and SS2, respectively, as indicated by the time of every measurement (UTC) reported above each plot. (a) Fourier power spectra, calculated as sum of square amplitudes. The significance levels, determined as explained in the text, are reported. (b) The wavelet-integrated power, calculated as sum of square amplitudes, is reported on the right ordinate, while on the left ordinate and abscissa the longitude and the wavenumber are respectively reported. 

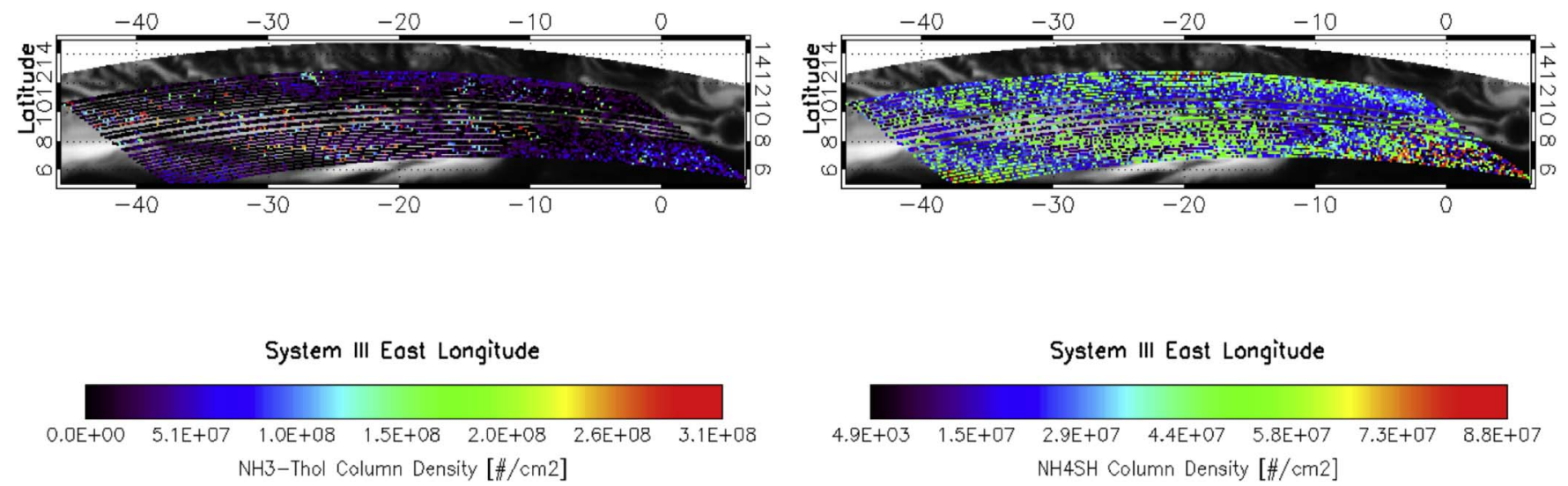

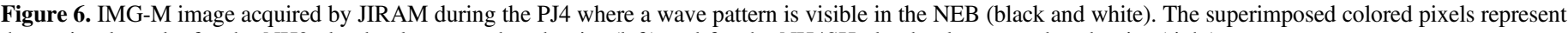
the retrieval results for the NH3 cloud column number density (left) and for the NH4SH cloud column number density (right).

(background power decreasing with increasing frequency/ wavenumber). For example, a 95\% peak-based critical limit implies that in one out of twenty random noise signals, the largest peak would reach this height by chance. Here we consider reliable those peaks overcoming a confidence level of $90 \%$, equivalent to a $10 \%$ significance (one out of ten). Besides the Fourier analysis, we carried out a double integration on the interpolated wavelet spectrum surface across all the wavenumbers and on the longitude range of each single data set. The results of the Fourier analysis and of this double integration, very similar to a smoothed FFT, are reported for each signal in Figure 5 (FFT in panel (a) and wavelet analysis in panel (b)). In Figure 5 from top to bottom, the plots refer to SS1 and SS2, respectively, as indicated by the time of every measurement (UTC) reported above each plot. The Fourier power spectra and wavelet-integrated power have been calculated as the sums of square amplitudes (PSD SSA).

The two methods reach very similar results. However, it is the wavelet power spectrum that gives the indication of which wavenumber carries most of the energy, on average. In the eastern region, a wavenumber of $0.65(\sim 1900 \mathrm{~km})$ prevails, while in the westward region, a contribution from $0.89(\sim 1400 \mathrm{~km})$ wavenumber appears. This westward value is in good agreement with that found in Simon et al. (2018) and Fletcher et al. (2018).

\subsection{Spectrometer Data from PJ4}

The retrieval results demonstrated a sufficient sensitivity (uncertainties $<50 \%$ ) in the spectral range $4.4-5 \mu \mathrm{m}$ only for the column density of the tholin-coated ammonia ice cloud and of the ammonium hydrosulfide cloud, as shown in Figure 6. In fact, although the retrieval code includes the cloud peak altitude, the ammonia relative "humidity" and the deep mixing ratio as free parameters, the spectral retrieval between 4.5 and $5.0 \mu \mathrm{m}$ does not provide sufficient constraints for these quantities in the region under study.

In order to highlight the wave patterns in the distribution of parameters we retrieved, we selected the one SPE slit crossing the wave front and another SPE slit crossing a wave-free region (Figure 2). Thus, we obtained longitudinal profiles of the retrieved parameters for fixed latitudes around $9^{\circ} \mathrm{N}$ and $10^{\circ} \mathrm{N}$ and east longitudes between $-15^{\circ}$ and $-10^{\circ}$ (Figure 7).

The analysis suggests the presence of two different perturbed clouds: the $\mathrm{NH}_{3}$ cloud at lower pressure levels and the $\mathrm{NH}_{4} \mathrm{SH}$ cloud at higher pressure levels. The longitudinal profiles across the wave front show clear wave patterns on both clouds with respect to the ones across the wave-free region. The latter is characterized by no wave patterns in the cloud densities. Moreover, this region is cloudier in the upper atmosphere having higher mean values for the $\mathrm{NH}_{3}$ cloud and lower mean values for the $\mathrm{NH}_{4} \mathrm{SH}$ cloud (Figure 7). In each case, the FWHM of the wave is of the order of 0.5 . The longitudinal positions of the wave peaks are slightly shifted for the two clouds densities. The wave peaks for the $\mathrm{NH}_{4} \mathrm{SH}$ cloud are mainly in agreement with the M-band integrated spectral radiances, whereas we observe a shift westward in longitude for the $\mathrm{NH}_{3}$ cloud profile. Although the wave amplitudes ( $>50 \%$ and $33 \%$ for $\mathrm{NH}_{3}$ and $\mathrm{NH}_{4} \mathrm{SH}$, respectively) are similar for the two clouds, we can retrieve the wave pattern for the ammonium hydrosulfide cloud with a higher accuracy, as the thermal spectral range we used is more sensitive to the largest particles composing this deep cloud. A more complete sounding of Jupiter's atmosphere, up to the stratosphere, requires the simultaneous analysis of radiances at other spectral regions. Unfortunately, as mentioned above, the JIRAM measurements are tied to the Juno orbit, which makes our observations available only in the night side, where only the thermal radiance comes from the planet.

\section{Discussion}

\subsection{Signal Processing Results}

The cloud crests of the wave pattern in the images acquired on the evening of 2017 July 10 (Figure 1 right panel) present changes in orientation and position with respect to those acquired 10 hours before (Figure 1 left panel). In panels (a) and (b) of Figure 8, some of the crests have been highlighted on the morning (08:35) and evening (18:42) geo-referenced images. The same images, in panel (c), are superimposed in an RGB color composition where the thinner clouds from the morning acquisition are in red and the ones from evening acquisition are in cyan. This rendering highlights the main feature displacements and changing shape of the cloud pattern, as highlighted in the appearance of the anti-cyclone (white circle). The wind field has also been derived (panel (d)) by the method described from Grassi et al. (2018). There, the wind vectors give the direction of the displacement of cloud features. All these renditions show that the wave patterns imaged in SS1 appear to be subject to variations in shape and orientation. This 

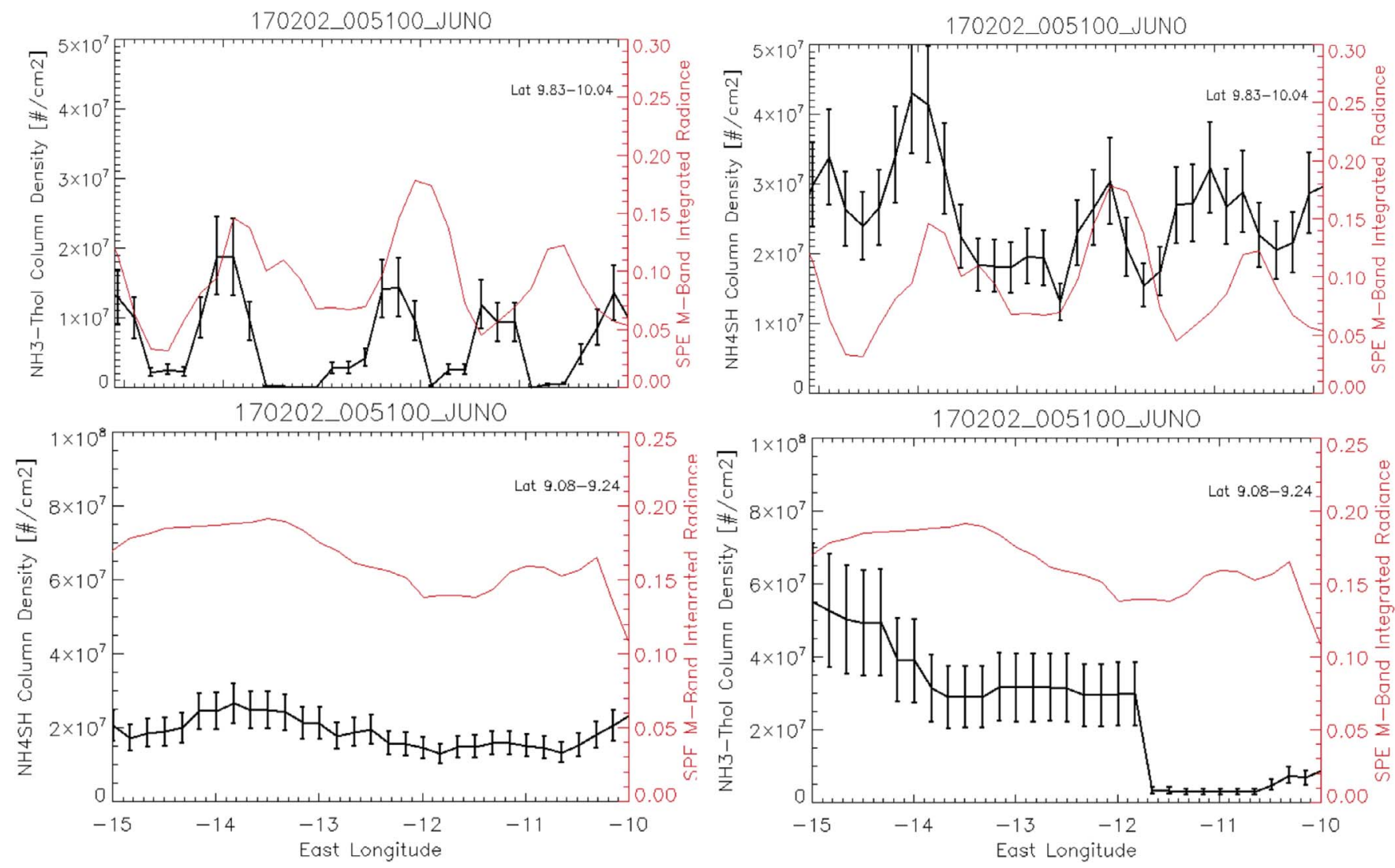

Figure 7. Longitudinal profiles for the NH3-tholin cloud (top left) and for the NH4SH cloud (top right) column number densities along a SPE slit crossing the wave front between $9.83 \mathrm{~N}$ and $10^{\circ} .4 \mathrm{~N}$ in latitude and between $-15^{\circ}$ and $-10^{\circ}$ east. The bottom panels represent the same quantities but for a SPE slit crossing a close wave-free region between $9.08 \mathrm{~N}$ and $9.24 \mathrm{~N}$.The black and red curves represent the retrieved parameters and the SPE M-band integrated radiance, respectively. Uncertainties are estimated as described in Sindoni et al. (2017).

is possibly due to interactions with the cyclones' and anticyclone's circulations, until the waves break down into turbulence, probably influenced by the shear of adjacent layers.

Looking at the first three rows from the top of Figure 5(a) in Section 5.1, the Fourier processing applied to SS1 produces power spectra, suggesting a progressive decline in the energies involved in the periodic component of the process, together with a slow transition toward higher wavenumbers, namely corresponding to a progressive reduction in the wavelengths, moving from east to west. The Fourier analysis of SS2 (second three rows from the top of Figure 5(a)) applies to the region covered by the first two SS1 plots (as can be seen by examining the longitudes reported on the left ordinate of the wavelet plots in Figure 5(b)). In this context, the SS2 analysis agrees with the SS1 one, both in terms of energies and wavenumbers of significant peaks, confirming the visual result of Figures 8(a) and (b).

Wavelet continuous transform applied to SS1 and SS2 signals give us a map of the power spectral densities in form of contour plots (Figure 10 in the Appendix). We emphasize that such an analysis is different than identifying a wavenumber from Fourier analysis, because the Fourier method only retrieves a space-invariant wave harmonic over the chosen space range. Such a retrieved wave is globally distributed and has no spatial information about the physical wave. With the wavelet transformation, we can instead localize the wave (when its wavenumber is known) within the spatial domain (Torrence \& Compo 1998).
An issue to consider in interpreting the wavelet map is the complexity of the cloud pattern, or the modulation in wavenumber in the SS1 and SS2 signals: it is not only the single wave visible in the images, but the result of many eddies overlapping, more or less permanent. We filtered all the contributions with wavenumbers less than 0.5 , but our sampling rate permits us to consider contributions up to 1.25 (Figure 5). Thus, we explain the positions of peaks in the power spectral density map of Figure 5(b) as sites where we can expect some reinforcement due to various physical mechanisms, such as wave-mean, wave-wave or wave-turbulence interactions, as well as wave breaking and/or reflection.

A comparison of the morning acquisitions at 09:40 and the respective wavelet transform is shown in Figure 9. Here the wavelet transform is reported as a contour plot overlapped to the image for corresponding longitude values. The ordinate values of the wavelet transform, originally in wavenumber, have been adapted to fit the latitude ordinate of the underlying map and located around the $15^{\circ}$ latitude of reference. In Figure 9, we see that some of the principal power peaks of the wavelet contour plot are located near to a mature cyclone $\left(15^{\circ} \mathrm{E}\right)$, one at an early stage $\left(15^{\circ} \mathrm{W}\right)$ and near to an anticyclone $\left(40^{\circ} \mathrm{W}\right)$. This result seems consistent with a scenario of waves generated by deep moist convection or, alternately, with an interaction between wave-background dynamics.

\subsection{Wave Types}

Waves detected on several Voyager images show a distinct class of patterns in the form of linear trains in the uppermost 


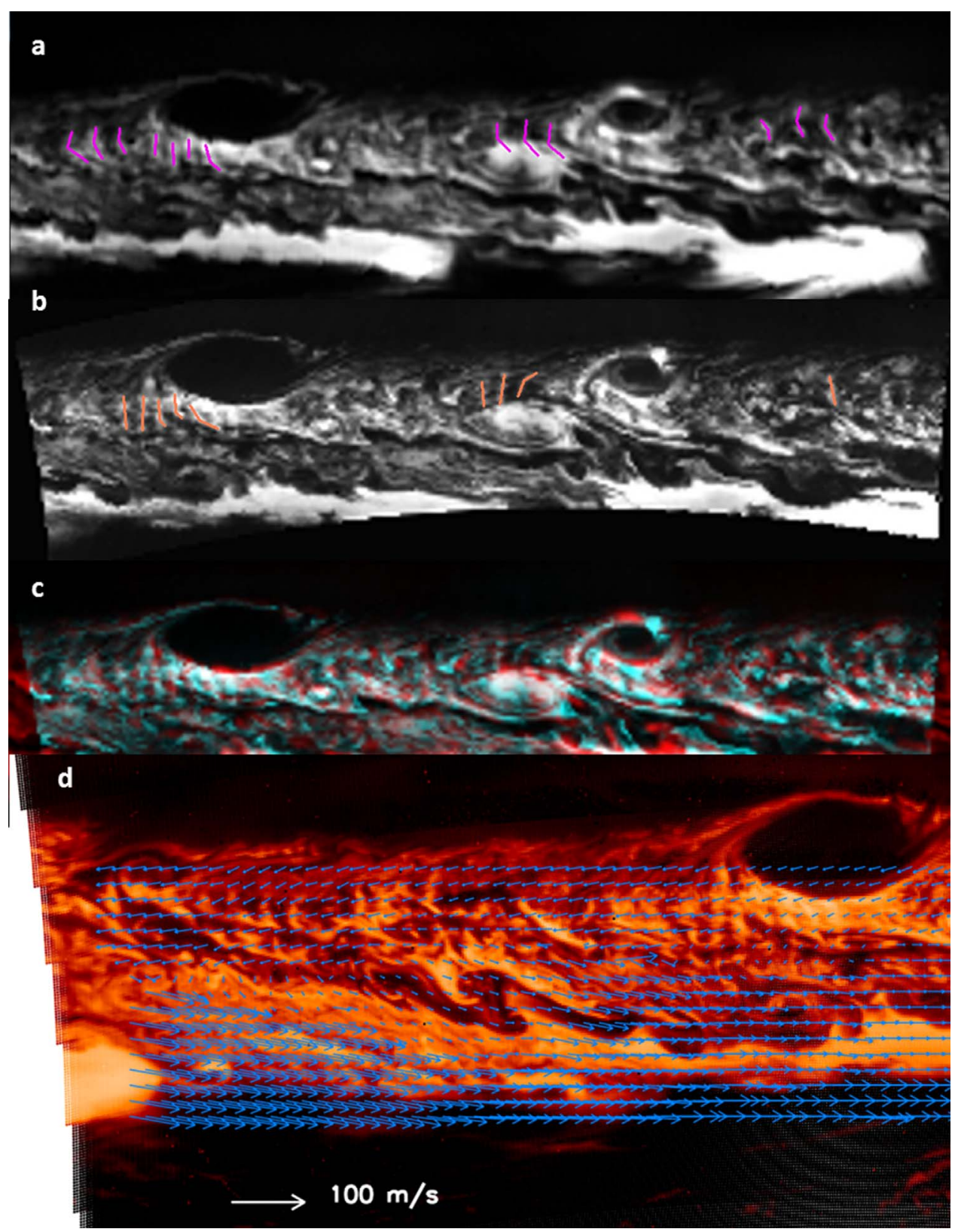

Figure 8. Detail of the wave region before and after 10 hours lapse. In panels (a) and (b), some of the most evident troughs have been underlined in magenta (morning) and orange (evening). In (b), some features have been lost, suggesting a breakdown of the wave pattern (right of image), while some other looks warped, probably due to the interaction with the surrounding mean (center and left of the image). In (c), the two images are stacked in an RGB color composition (see the text). Panel (d) is overlapped with the horizontal wind field, determined by the Grassi et al. (2018) algorithm.

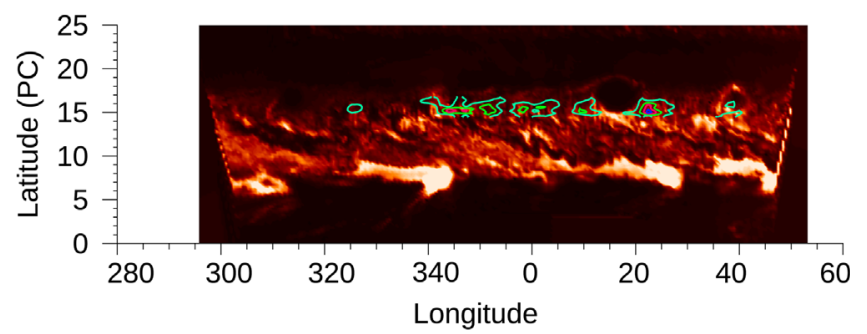

Figure 9. Morning JIRAM acquisition with corresponding wavelet transform map overlapped. In these images' composition, the vertical dimension of the wavelet map (originally in wavenumber) has been adapted to the latitude ordinate of the JIRAM image. The power spectrum contour plot is centered around $15^{\circ}$, that is the latitude of reference. ammonia clouds with a characteristic horizontal wavelength of about $300 \mathrm{~km}$ and a small dispersion on the order of $\pm 20 \%$ (Flasar \& Gierasch 1986). They were mainly observed near local maxima of the zonal wind near the equator with crests oriented north-south, perpendicular to the zonal flow. These features were interpreted by Flasar \& Gierasch (1986) as ducted gravity waves trapped in a layer underneath the ammonia cloud. The phase velocity could not be measured. An alternate model (Bosak \& Ingersoll 2002), involving shear instability (KH or Kelvin-Helmholtz instability), could account for the observed wavelength provided the shear layer thickness was set to $45 \mathrm{~km}$, which is comparable to the shear layer measured by the Galileo probe (Seiff et al. 1997). For shear 
instability, the pattern speed was intermediate between the speeds at the top and bottom of the layer.

Cameras on the New Horizons spacecraft also saw $300 \mathrm{~km}$ waves and were able to measure the phase speed (Simon et al. 2015a), which turned out to be to the east and $90 \mathrm{~m} \mathrm{~s}^{-1}$ faster than the local zonal flow. This high speed was not predicted by either of the two earlier models (Flasar \& Gierasch 1986; Bosak \& Ingersoll 2002), but these waves are not the same in appearance, being centered symmetrically on the equator and extending over a much larger longitude range. These waves are instead consistent with a Kelvin wave.

A number of atmospheric perturbations produce wave-like structures. Gravity waves, which require a source or obstacle and involve gravity as restoring force, are the most likely class of wave phenomena in planetary atmospheres. By inducing fluctuation on both temperature and density fields, they can be detected by means of several different techniques (e.g., Altieri et al. 2014 and references therein for Terrestrial Planets; Young et al. 1997; Arregi et al. 2009 for Jupiter). Conversely, other phenomena, such as shear instabilities, do not need a source. They are self-exciting once the flow becomes unstable. KelvinHelmholtz "billow" clouds originate in an atmosphere under these conditions. They are common on Earth and have been observed on Saturn (http://ciclops.org/view/479/Goo-GooGa-Joob). Moist convection triggers a broad spectrum of gravity waves via latent heat release and interactions with the mean flow (see Figure S4 in the Supporting Information of Miller et al. 2015). Gravity waves could be generated by this mechanism in Jupiter's atmosphere. However, the extremely long wavelength, the linear structure, and the coherence over large distances with respect to already known cases implies that these features are unique and merit caution in our assignment of the class of wave.

Even if one is fairly sure that one is seeing a gravity wave, the interpretation can be ambiguous. Dark rings from the Shoemaker-Levy 9 impact sites propagated outward at speeds of $\sim 450 \mathrm{~m} \mathrm{~s}^{-1}$ (Hammel et al. 1995). This was interpreted as a ducted gravity wave trapped at the top by the highly stable stratosphere and trapped at the bottom by the adiabatic interior (Ingersoll \& Kanamori 1995). The tropospheric weather layer in between had to be highly stable to match the high speeds, which was taken as evidence for an extremely high water abundance. However, a later model (Walterscheid et al. 2000) showed that waves propagating upward and outward in the stable stratosphere could also match the observed speed. The loss of wave energy due to upward propagation did not prevent the wave from spreading horizontally as a ring in the clouds.

The waves detected in JIRAM and ground-based data (Fletcher et al. 2018) have a 1300-1600 wavelength, which is very different from the $300 \mathrm{~km}$ wavelength observed in the Voyager images. Typical wavelengths of $1200 \mathrm{~km}$ were first detected in visible light in Hubble Space Telescope images (Simon et al. 2015b). Those authors interpreted the waves as a baroclinic instability associated with cyclone formation near $16^{\circ} \mathrm{N}$ latitude. The JIRAM waves are sometimes located close to cyclonic features, but they are also detected in anticyclonic regions and in intermediate regions. Occurrences of gravity wave $(\mathrm{GW})$ and inertia-gravity wave (IGW) are investigated by Fletcher et al. (2018) and Simon et al. (2018). Assuming CIRSderived thermal structure above the 1-bar pressure level, they find that IGWs are unable to propagate vertically while GW vertical propagation would be favored near 400-600 mbar with a confined vertical wavelength. However, the true nature of the waves remains elusive due to the difficulties in quantifying the unknown parameters. In particular, it not possible to uniquely identify the wave type without knowledge of vertical structure and phase velocity when the only observations are periodic rows of clouds of a given wavelength. Fletcher et al. (2018) and Simon et al. (2018) found that the region of the NEB studied exhibits the necessary conditions to violate a range of instability criteria, favoring wave genesis at the observed latitude.

\subsection{Spectroscopic Analysis Results}

The spectroscopic analysis of the wave observed during the PJ4 suggests that, in this case, the wave phenomenon occurs at different atmospheric altitudes and so affects both the $\mathrm{NH}_{4} \mathrm{SH}$ and the $\mathrm{NH}_{3}$ clouds. Moreover, we observe a shift in the longitudinal position of the crests of the two clouds (Figure 8). The first and deeper one (at about 1.5-2 bar) is further east with respect to the second and higher one (at about $1-0.5$ bar). In particular, we estimated the longitudinal shift using the Pearson correlation. We synthetically shifted the longitudinal position of the $\mathrm{NH}_{3}$ cloud respect to the $\mathrm{NH}_{4} \mathrm{SH}$ one between -0.5 and 0.5 with an accuracy of 0.001 and interpolated the longitudinal profile on the shifted longitude grid. Thus, we identified the maximum correlation coefficient (0.776) for a longitudinal shift of 0.071 westward on the entire wave front (longitudes between $15^{\circ} \mathrm{W}$ and $10^{\circ} \mathrm{W}$ ). At the latitudes where we observe the wave front (around $10^{\circ} \mathrm{N}$ ), this shift corresponds to about $90 \mathrm{~km}$. If we consider the wave crests separately, we find different shifts. The crests around $14^{\circ} \mathrm{W}, 12^{\circ} \mathrm{W}$ and $11^{\circ} \mathrm{W}$ show a longitudinal shift of $-0.023(28 \mathrm{~km}), 0^{\circ} .116(142 \mathrm{~km})$ and $0.298(366 \mathrm{~km})$ westward with correlation coefficients of $0.987,0.941$ and 0.861 , respectively. Therefore, the crests in the $\mathrm{NH}_{3}$ cloud are closer to each other than the ones in the $\mathrm{NH}_{4} \mathrm{SH}$ cloud.

\subsection{Westward Tilt}

The waves are observed to be lined up with the zonal velocity and are tilted westward with increasing altitude, with about a $10 \%\left(\sim 36^{\circ}\right)$ phase lag of the wave at the level of the ammonia clouds relative to that at the $\mathrm{NH}_{4} \mathrm{SH}$ cloud. Gravity waves flowing over an obstacle and baroclinic instability both have tilted phase lines, but for different reasons. For a baroclinically unstable zonal flow, the westward tilt arises when the wind becomes stronger and more eastward with altitude. Then the fastest-growing disturbance is a wave that varies periodically with longitude and whose phase lines slope upward to the west (Holton \& Hakim 2013). For gravity waves, the westward tilt occurs even when there is a uniform (unsheared) flow, provided it is relative to the obstacle that is acting as the wave source (Holton \& Hakim 2013). This is somewhat counterintuitive, since one might expect the wake from the obstacle to trail off downstream to the east. The westward tilt arises because the group velocity of the gravity wave must be upward when the source is at the ground.

In both cases, there has to be an eastward flow to get a westward tilt, and that seems to contradict the fact that the flow is westward from $13^{\circ} .6 \mathrm{~N}$ to $17^{\circ} .1 \mathrm{~N}$ planetocentric latitude (Limaye 1986), which is where the waves are observed. However, that latitude band is characterized by strong wind shear, as deduced from the thermal wind equation and Voyager 
observations of temperature at the 270-mbar level (Gierasch et al. 1986). Analysis of Cassini CIRS data show that the wind shear can be time variable, particularly near the equator, but at these latitudes, the wind becomes more eastward with altitude at a rate of $20 \mathrm{~m} \mathrm{~s}^{-1}$ per scale height (Simon-Miller et al. 2006). The Galileo probe also observed winds decreasing with altitude from the 4-bar level up at least to the 0.5-bar level (Seiff et al. 1997). Therefore, for the NEB both the baroclinic waves and the gravity waves from a deep source will have a westward tilt. Here "deep" simply means below the level where the waves are observed. Although JIRAM sees deeper than Voyager IRIS or Cassini CIRS, these qualitative statements still hold if the wind shear persists down to the $\mathrm{NH}_{4} \mathrm{SH}(\sim 2.5-$ bar) level.

\section{Summary and Conclusions}

We analyzed two different observations taken at different times on the NEB. It was necessary to divide the analysis into two parts, one using the images of PJ7 for the wave characterization from the horizontal patterns, and the other using the spectral data of PJ4, to determine the wave vertical structure using a radiative-transfer model. Unfortunately, the spectral coverage obtained during the PJ7 pass was not sufficiently detailed to assure a good retrieval for identifying the wave structures we observed in the NEB. On the other hand, the images from PJ4 only partially covered the NEB $15^{\circ}$ region where the $\mathrm{PJ} 7$ waves were observed, but they gave very good coverage of the NEB $10^{\circ}$ region where other waves could also be seen. The PJ7 images, analyzed by Fourier and wavelet transforms, are consistent with wave packets having wavelengths generally decreasing, moving westward, and ranging from 1900 to $1400 \mathrm{~km}$. The non-stationary components, exploited by the wavelet analysis, point to a source or interaction mechanism near cyclones and anticyclones.

The spectral contribution determines the waves' vertical position. The radiance at the wavelength of $5 \mu \mathrm{m}$ comes mostly from atmospheric pressure levels greater than 1 bar. The presence of wavy structures in the aerosol present at pressure levels smaller than 1 bar can modulate the $5 \mu \mathrm{m}$ radiance coming from the lower levels of the atmosphere. However, the spectral data can contribute significantly in characterizing the actual vertical positions of the wavy structures. In fact, in the spectral analysis, the contribution from the different clouds and/or aerosol can be decoupled to identify the contribution of the different components. Figure 8 shows that the $\mathrm{NH}_{3}$ cloud column number density and the $\mathrm{NH}_{4} \mathrm{SH}$ cloud are present at both levels even if the higher cloud perturbations are shifted by about $0^{\circ} .3$ westward on average, possibly due to the different wind speeds between the two pressure levels.

We cannot definitely state that the spectral analysis done for PJ4 is valid also for PJ7 case and that those waves also can occur simultaneously at both $\mathrm{NH}_{3}$ and $\mathrm{NH}_{4} \mathrm{SH}$ levels, because we did not have spectral coverage to prove it. However, the theoretical considerations given in the discussion and the results from the $\mathrm{PJ} 4$ data strongly suggest that the wavy perturbation of the NEB could also be present at deeper pressure levels of 1.5-2 bar where $\mathrm{NH}_{4} \mathrm{SH}$ clouds are putatively present (Sindoni et al. 2017). In summary, we have been able to characterize the appearance of the observed waves, quantify the variation in the $\mathrm{NH}_{3}$ and $\mathrm{NH}_{4} \mathrm{SH}$ column densities induced by wave propagation, and advance some hypothesis on their vertical location. We also were able to identify the region showing the largest energies involved in the process. On the other hand, additional parameters needed to constrain the nature of the wave are missing. It could be a gravity wave, a baroclinic instability, or possibly a $\mathrm{KH}$ instability if the shear layer is deep enough. Our caution is merited because these data do not constrain the properties of the medium where the wave occurs-its vertical stratification, its thickness, the degree of wind shear, and so on. In order to identify the type of waves, other crucial information is needed, such as phase velocity and lifetime. A more conclusive assessment of the nature of the observed periodic patterns would allow probing below the surface (1-bar level), as is done with seismic waves.

The JIRAM project is funded by the Italian Space Agency (ASI). In particular, this work has been developed under the ASI-INAF agreement n. 2016-23-H.0. A portion of this work was funded by NASA through the Juno mission, a portion of which was distributed to the Jet Propulsion Laboratory, California Institute of Technology.

\section{Appendix A IMG-M Data Analysis}

Fourier transform (Bendat \& Piersol 1986) is a powerful tool for analyzing the components of stationary signals, and in recent years wavelet transform has become a valuable analysis tool to complement traditional spectral analyses, localizing in the inverse space (time or space) the contribution under analysis (frequency or wavenumber). The wavelet transform method (Graps 1995; Resnikoff \& Wells 1998) also allows us to analyze components of non-stationary signals. In other words, they are well-suited to identify possible changes in wave amplitudes and/or wavenumber/frequency related to physical mechanisms (for example wave-wave or waveturbulence interactions). The Fourier method allows us to search for the highest wavenumber contributions, whereas the use of wavelets principally is for determining the spatial characteristics and possibly breaking or interaction areas of the imaged mesoscale waves. Our spatially distributed data are transformed in a similar-wavenumber space, since the spatial coordinates of the series are in longitude degrees.

The wavelengths of the waves, found in field of the longitudes, have been then transformed into kilometers to give a measurement in terms of physical distance. To determine the horizontal wavelength of the wave, the distance in kilometers is recovered after the spectral analysis by calculating how many kilometers correspond to one longitude degree at $15^{\circ} \mathrm{N}$ latitude by applying the known relationship: 1 longitude degree $=2^{*}$ $\pi^{*} R_{\mathrm{e}} / 360^{\circ}$, that works for the circle maximum at the equator, adapted to a different latitude for an oblate body.

In the wavelet analysis, we have used a low value of scale parameter $(s=8)$ to favor a better spatial localization against a high wavenumber resolution, granted anyway by the Fourier analysis. In fact, the localization is more or less accurate depending on the scale parameter value that settles on the scale length.

\section{Appendix B Spectral-data Analysis}

The spectral range $4.4-5 \mu \mathrm{m}$ is more sensitive to the putative deep ammonium hydrosulfide $\left(\mathrm{NH}_{4} \mathrm{SH}\right)$ cloud than the reflected-sunlight range, since the sensitivity to small particles 

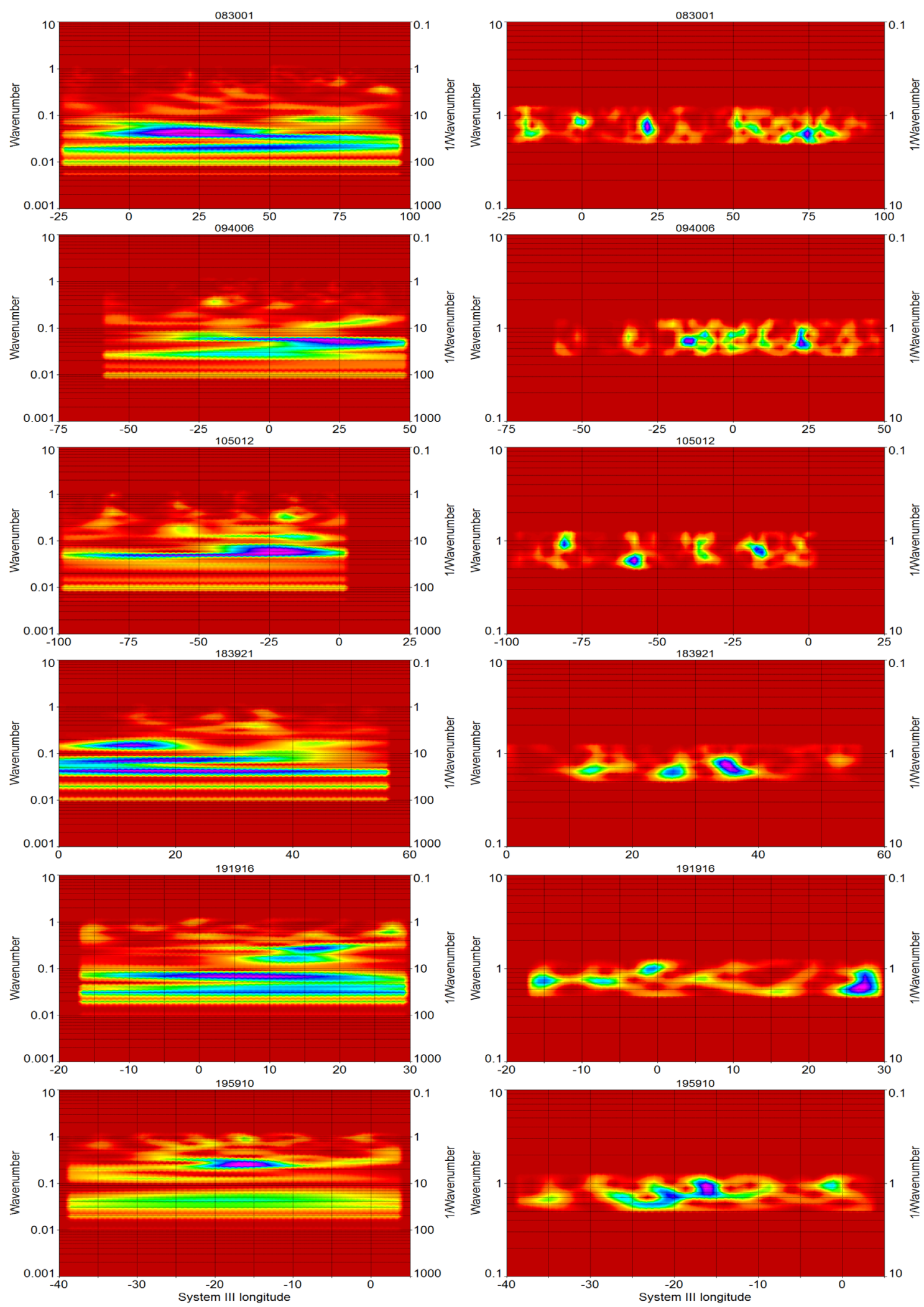

Figure 10. Wavelet transforms of the signal samples extracted along the $15^{\circ} \mathrm{N}$ latitude from the images of Figure 1. Each image reports the UTC time of acquisition in the title. On the left and right ordinates, the wavenumber, in the sense of inverse of longitude degrees as explained in the paper, and the relative period are reported. The abscissa is in System III longitude, but visualized according to the west/east convention. Panel (a): transforms of the unfiltered signal samples. Components with period ranging from 20 to 100 longitude degrees $(\sim 25000-124,000 \mathrm{~km}$ ) are prevailing. Panel (b): transforms of the signal samples after high-pass filtering (cutoff 0.5 ). A quasi-continuous zone of components covering the range of sampling is magnified after the filtering. The low value of scale parameter used in the analysis gives a detailed localization of the signal non-stationary components. 
located in the high troposphere or in the stratosphere, like $\mathrm{NH}_{3}$ ice clouds and hazes, significantly decreases with the wavelength. The atmospheric model used (Grassi et al. 2010; Sindoni et al. 2017) takes into account the gaseous opacities of $\mathrm{CH}_{4}, \mathrm{H}_{2} \mathrm{O}, \mathrm{NH}_{3}, \mathrm{PH}_{3}$ and the collision-induced absorptions by $\mathrm{H}_{2}-\mathrm{H}_{2}, \mathrm{H}_{2}-\mathrm{He}$, and $\mathrm{H}_{2}-\mathrm{CH}_{4}$. The temperature-pressure profile is derived from the Galileo Probe data (Seiff et al. 1998) and two tropospheric clouds and a stratospheric haze layer describe the atmospheric particulate components. The deepest $\mathrm{NH}_{4} \mathrm{SH}$ cloud is described by the optical properties suggested by Giles et al. (2015), in which they assumed a single-scattering albedo $\omega=0.9$ and asymmetry factor $g=0.7$ for the HenyeyGreenstein phase function in the entire spectral range of our interest. In the assumed atmospheric scenario, the high tropospheric cloud is composed by $\mathrm{NH}_{3}$ ice with a tholin coating and a stratospheric $\mathrm{N}_{2} \mathrm{H}_{4}$ haze is considered. The properties of these two aerosol components are described in detail in Sindoni et al. (2017).

The analysis procedure retrieves the following parameters for each pixel, starting from the a priori values:

(1) the column number density for the $\mathrm{NH}_{3}$ cloud;

(2) the peak altitude of the $\mathrm{NH}_{3}$ cloud;

(3) the column number density for the $\mathrm{N}_{2} \mathrm{H}_{4}$ haze;

(4) the peak altitude of the $\mathrm{N}_{2} \mathrm{H}_{4}$;

(5) the column number density for the $\mathrm{NH}_{4} \mathrm{SH}$ cloud;

(6) the peak altitude of the $\mathrm{NH}_{4} \mathrm{SH}$ cloud;

(7) the deep mixing ratio of gaseous ammonia;

(8) the relative "humidity" of gaseous ammonia.

The parameters (5) and (6) were added to the retrieval algorithm described in Sindoni et al. (2017) in order to take into account the thermal spectral range sensitivity to the $\mathrm{NH}_{4} \mathrm{SH}$ deep cloud. Other atmospheric quantities were fixed to their a priori values.

For each parameter, we filtered the retrieved values, discarding those out of the $99 \%$ confidence level for the statistical distribution of the parameter population on the entire retrieved data set. This statistical filter, in fact, rules out the retrieved values with a high probability of being nonphysical, even if they provide a good numerical fit.

\section{ORCID iDs}

A. Adriani (1) https://orcid.org/0000-0003-4998-8008

M. L. Moriconi (i) https://orcid.org/0000-0003-2609-2620

F. Altieri (1) https://orcid.org/0000-0002-6338-8300

G. Sindoni (ㄷ) https://orcid.org/0000-0002-3348-7930
L. N. Fletcher (1) https://orcid.org/0000-0001-5834-9588

A. A. Simon (ㄴ) https://orcid.org/0000-0003-4641-6186

\section{References}

Acton, C. H. 1996, P\&SS, 44, 65

Acton, C. H., Bachman, N., Semenov, B., \& Wright, E. 2018, P\&SS, 150, 9

Adriani, A., Filacchione, G., Di Iorio, T., et al. 2014, SSRv, 213, 393

Altieri, F., Migliorini, A., Zasova, L., et al. 2014, JGRE, 119, 2300

Arregi, J., Rojas, J. F., Hueso, R., \& Sánchez-Lavega, A. 2009, Icar, 202, 358

Atreya, S. K., Rojas, J. F., Hueso, R., \& Sanchez-Lavega, A. 2005, P\&SS, 53, 498

Bendat, J. S., \& Piersol, A. G. 1986, Random Data: Analysis and Measurement Procedures (New York: Wiley), 566

Bjoraker, G. L., Wong, M. H., De Pater, I., \& Adamkovics, M. 2015, ApJ, 810,122

Bosak, T., \& Ingersoll, A. P. 2002, Icar, 158, 401

Flasar, F. M., \& Gierasch, P. J. 1986, JAtS, 43, 2683

Fletcher, L. N., Melin, H., Adriani, A., et al. 2018, AJ, 156, 67

Fletcher, L. N., Orton, G. S., Sinclair, J. A., et al. 2017, GeoRL, 44, 7140

Gierasch, P. J., Conrath, B. J., \& Magalhaes, J. A. 1986, Icar, 67, 456

Giles, R. S., Fletcher, L. N., \& Irwin, P. G. J. 2015, Icar, 257, 457

Graps, A. 1995, ICSEn, 2, 50

Grassi, D., Adriani, A., Moriconi, M. L., et al. 2010, P\&SS, 58, 1265

Grassi, D., Adriani, A., Moriconi, M. L., et al. 2018, JGRE, 123, 1511

Grassi, D., Adriani, A., Mura, A., et al. 2017, GeoRL, 44, 4615

Hammel, H. B., Beebe, R. F., Ingersoll, A. P., et al. 1995, Sci, 267, 1288

Holton, J. R., \& Hakim, G. J. 2013, An Introduction to Dynamic Meteorology (5th ed.; Waltham, MA: Academic, Elsevier)

Hunt, G. E., \& Muller, J. P. 1979, Natur, 280, 778

Ignatiev, N. I., Grassi, D., \& Zasova, L. V. 2005, P\&SS, 53, 1035

Ingersoll, A. P., \& Kanamori, H. 1995, Natur, 374, 706

Kataza, H., Okamoto, Y., Takubo, S., et al. 2000, Proc. SPIE, 4008, 1144

Lagage, P. O., Pell, J. W., Authier, M., et al. 2004, Msngr, 117, 12

Limaye, S. S. 1986, Icar, 65, 335

Lin, Y.-L. 2007, Mesoscale Dynamics (Cambridge: Cambridge Univ. Press)

Miller, S. D., Straka, W. C., III, Yue, J., et al. 2015, PNAS, 112, E6728

Resnikoff, H. L., \& Wells, R. O., Jr. 1998, Wavelet Analysis, The Scalable Structure of Information (New York: Springer)

Rodgers, C. D. 2000, Inverse Methods for Atmospheric Sounding: Theory and Practice (Singapore: World Scientific)

Seiff, A., Blanchard, R. C., Knight, T. C. D., et al. 1997, Natur, 388, 650

Seiff, A., Kirk, D. B., Knight, T. C. D., et al. 1998, JGR, 103, 22857

Simon, A. A., Hueso, R., Iñurrigarro, P., et al. 2018, AJ, 156, 79

Simon, A. A., Li, L., \& Reuter, D. C. 2015a, GeoRL, 42, 2612

Simon, A. A., Wong, M. H., \& Orton, G. S. 2015b, ApJ, 812, 55

Simon-Miller, A. A., Conrath, B. J., Gierasch, P. J., et al. 2006, Icar, 180, 98

Sindoni, G., Grassi, D., Adriani, A., et al. 2017, GeoRL, 44, 4660

Torrence, C., \& Compo, G. P. 1998, BAMS, 79, 61

Walterscheid, R. L., Brinkman, D. G., \& Schubert, G. 2000, Icar, 145, 140

West, R. A., Baines, K. H., James, F. A., et al. 2004, in Jupiter. The planet, satellites and magnetosphere, ed. F. Bagenal et al. (Cambridge: Cambridge Univ. Press), 79

Young, L. A., Yelle, R. V., Young, R., Seiff, A., \& Kirk, D. B. 1997, Sci, 276,108 